\title{
Empirical Analysis of Influential Factors for the Quality Accumulation of Industrial Cluster's Human Capital
}

\author{
Siwei Sun ${ }^{1, a}$,Qianyun Sun², b \\ ${ }^{1}$ School of Management, Xiamen University, Zhejiang Ningbo 315300, China; \\ ${ }^{2}$ College of Applied Science and Technology, Hainan University, Hainan Danzhou 571737, China. \\ a2253900501@qq.com, b83073210@qq.com
}

\begin{abstract}
This paper, based on the theory of industrial cluster and human capital, first constructs the panel data model of industry cluster's human capital accumulation from the cluster's external environment and their own factors, and then makes the empirical analysis for the national's 35 software parks with the statistical data. The results show that the accumulation of human capital quality of the industrial cluster has Matthew Effect, the region's economic environment and education environment has a significant positive effect on the quality of the cluster's human capital accumulation while the cluster scale has the negative effect, and the other factors were not significant.
\end{abstract}

Keywords: Industrial cluster; quality of human capital; panel data model.

\section{产业集群人力资本质量积累影响因素实证分析}

\author{
孙思为 ${ }^{1}$, 孙倩芸 ${ }^{2}$ \\ 1. 厦门大学 管理学院, 浙江 宁波 中国; \\ 2. 海南大学 应用科技学院, 海南 儋州 中国
}

摘要: 本文以产业集群与人力资本理论为基础, 首先从集群外部环境和集群自身因素两大方 面构建了集群人力资本质量积累的面板数据模型, 然后基于统计年鉴数据, 对国家火炬计划 软件产业基地中的 35 个软件园进行了实证分析。研究结果表明, 产业集群人力资本质量积累 存在马太效应, 集群所在地区的经济环境和教育环境正向显著影响集群人力资本质量积累水 平, 集群规模的影响方向则相反, 其他影响因素不显著。

\section{关键词：产业集群；人力资本质量；面板数据模型}

\section{1. 前言}

目前, 我国很多地区在不同产业形成了不同规模和水平的产业集群。对此, 已有学者从 经济学、管理学、社会学等多领域视角对集群现象进行了深入研究。但是针对产业集群人力 资本质量积累影响因素的系统研究还未开展。本文选取国家火炬计划软件产业基地中 35 个软 件园为集群分析对象, 寻求外部因素对集群人力资本质量积累的影响程度。

\section{2. 实证分析}

\section{1. 变量选取}

从企业角度考察人力资本, 可用企业总收入与总人数之比值来衡量一个企业的平均生产 率或人均产值 [1]。本研究前提假设集群内企业具有同质性, 把产业集群人均生产率来表示 并作为被解释变量。 
产业集群人力资本质量积累主要受集群外部环境和集群自身两大方面的影响。本文分别 从这两大因素构造模型解释变量。

本文将解释变量进行汇总, 得如下表 1 。

表1 产业集群人力资本质量积累影响因素解释变量列表

\begin{tabular}{|c|c|}
\hline 变量 & 定义 \\
\hline \multicolumn{2}{|l|}{ 产业集群外部环境因素 } \\
\hline 人均GDP （pgdp） & Ln（人均地区生产总值） \\
\hline 职工平均工资（sal） & Ln（职工平均工资） \\
\hline 人均教育支出（edu） & Ln（教育支出/当年总人口） \\
\hline 医生数/万人（doct） & 每万人拥有的医生数量（无量纲） \\
\hline 人均城市道路面积（road） & 市辖区人均道路面积（平方米） \\
\hline 人均绿化面积 (grn) & 市辖区人均绿化面积（平方米） \\
\hline \multicolumn{2}{|l|}{ 产业集群自身因素 } \\
\hline $\begin{array}{l}\text { 政府对集群的支持率: } \\
\text { 政府投入 (gov) }\end{array}$ & $\begin{array}{c}\text { （政府部门资金+地方政府资金+减免税总额）/(实 } \\
\text { 际上缴税金+减免税总额）(\%) }\end{array}$ \\
\hline $\begin{array}{c}\text { 集群企业自身投入: } \\
\text { (clus) }\end{array}$ & 科技活动经费支出总额（千元） \\
\hline 产业集群规模（scal） & Ln (年末产业园总人数) \\
\hline
\end{tabular}

\section{2. 模型数据来源及说明}

本文选取国家火炬计划软件产业基地中 35个软件园2008-2011年四年的数据作为模型中 集群层面的数据，来源于《中国火炬统计年鉴》（2009-2012）［2］；选取《中国城市统计 年鉴》（2009-2012）［3］中35个软件园所在城市的数据，并对各地市职工平均工资和人均 教育支出均用城市居民消费以及人均GDP和人均生产率用工业生产者出厂价格指数按 2008 年 不变价进行平减处理。

\section{3. 模型选择}

本文在研究方法上把传统的静态面板与动态面板相结合, 并对实证结果进行比较分析。

2.3. 1 静态面板数据模型

（1）模型构建

以产业集群人力资本质量积累水平为被解释变量, 以表1中所列的变量为解释变量, 将面 板数据模型设为模型 1 的形式:

$$
\begin{aligned}
& y_{i t}=\alpha_{i}+\beta_{1} \ln p g d p+\beta_{2} \ln s a l+\beta_{3} d o c t+\beta_{4} \ln e d u+\beta_{5} g r n+\beta_{6} \text { road } \\
& +\beta_{7} \text { gov }+\beta_{8} \text { clus }+\beta_{9} \ln s c a l+u_{i}+\varepsilon_{i t}
\end{aligned}
$$

其中， $i=1 ， 2 \cdots, 35$ 代表第 $i$ 个截面观察单位， $t$ 代表时间序列观察值。 $y_{i t}$ 表示第 $i$ 软件 园、 $t$ 年的人力资本质量积累水平; $\beta_{k}(k=1,2, \cdots 10)$ 表示所有单位的斜率系数。假设个体 效应 $u_{i}$ 是常量, $u_{i}$ 代表恒常不变的影响产业集群人力资本质量积累的因素, 其它随时间而变 的因素作用归入随机项 $\varepsilon_{i t}$ 中, 此时模型为固定效应模型; 反之, 若个体效应 $u_{i}$ 是随机变量, 随机误差项则变为 $u_{i}+\varepsilon_{i t}$, 此时模型则为随机效应模型。考虑到变量 $p g d p$ (人均GDP) 和 $s a l$

(职工平均工资) 间可能存在多重共线性, 故本文先对这两个变量进行相关性分析, 结果发 现p值为 0.0000 , 相关系数为 0.9739433 , 说明两者存在强相关。故本文剔除变量 $p g d p$ 。模型 1 简化为模型 2 的形式。

$$
\begin{aligned}
& \ln y_{i t}=\alpha_{i}+\beta_{1} \ln s a l+\beta_{2} d o c t+\beta_{3} \ln e d u+\beta_{4} g r n+\beta_{5} \text { road } \\
& +\beta_{6} \text { gov }+\beta_{7} c l u s+\beta_{8} \ln s c a l+u_{i}+\varepsilon_{i t}
\end{aligned}
$$




\section{(2) 模型结果分析}

模型2混合回归、随机效应模型和固定效应模型估计结果。通过Hausman检验，得到p值为 0.0001, 故本文认为应该使用固定效应模型。由固定效应模型估计结果可知, 解释变量中职 工平均工资 $(s a l)$ 、人均教育支出 $(e d u)$ 、人均绿化面积 $(g r n)$ 和集群自身投入 ( clus) 正向影响产业集群人力资本质量积累, 集群规模 ( scal) 的影响方向则为负。职工平均工资 ( sal ) 代表了产业集群所在的地区的人均收入水平，该变量正向影响集群人力资本质量积累 水平。当集群所在城市的职工平均工资提高1个百分点时, 集群人力资本质量积累水平提高 0.4478 个百分点。人均教育支出 $(e d u)$ 代表了一个地区的教育投入水平，当人均教育支出 增加 1 个百分点时，产业集群人力资本质量积累水平提高 0.2288 个百分点。人均绿化面积

( grn ) 代表市政基础设施环境的一个方面, 该变量正向影响集群人力资本质量积累水平。 当集群所在城市的人均绿化面积增加 1 平方米, 集群人力资本质量积累水平提高 0.0293 个百分 点。集群自身投入 ( clus ) 正向影响集群人力资本质量积累水平。当集群自身投入增加1个百 分点时, 集群人力资本质量积累水平提高 0.1835 个百分点。集群规模 ( scal ) 负向影响集群 人力资本质量积累水平。当集群规模扩大 1 个百分点时, 集群人力资本质量积累水平下降 0.6761 个百分点。集群规模负向影响产业集群人力资本的质量积累水平。

2.3.2 动态面板数据模型

将滞后一期的产出作为解释变量引入方程, 模型如下:

$\ln y_{i t}=\alpha_{i}+\beta_{0} \ln y_{i t-1}+\beta_{1} \ln s a l+\beta_{2} d o c t+\beta_{3} \ln e d u+\beta_{4}$ grn $+\beta_{5}$ road

$+\beta_{6}$ gov $+\beta_{7}$ clus $+\beta_{8} \ln s c a l+u_{i}+\varepsilon_{i t}$

(模型3)

对于动态面板数据模型，其估计的前提是面板数据必须是平稳的，否则可能产生伪回归 的结果。因此, 本文检验动态面板数据的平稳性, 先估计模型, 再对估计后的残差进行检验, 若残差平稳, 则可推断出动态面板数据是平稳的。对模型 3 进行差分处理, 得到模型 4 :

$$
\begin{aligned}
& \Delta \ln y_{i t}=\beta_{0} \Delta \ln y_{i t-1}+\beta_{1} \Delta \ln s a l+\beta_{2} \Delta d o c t+\beta_{3} \Delta \ln e d u+\beta_{4} \Delta g r n+\beta_{5} \Delta \text { road } \\
& +\beta_{6} \Delta g o v+\beta_{7} \Delta c l u s+\beta_{8} \Delta \ln s c a l+u_{i}+\varepsilon_{i t}
\end{aligned}
$$

将包含水平值的原模型（模型3）和经差分处理后的模型（模型4）构成一个系统，在系 统的矩估计过程中, 解释变量的滞后值作为一阶差分方程的工具变量, 而解释变量一阶差分 的滞后值作为水平变量估计方程的工具变量。此方法纳入了所有的矩条件, 估计结果在统计 上也更加有效, 适合动态面板数据模型的估计。本文引入被解释变量 $\ln y$ 一期滞后值, 首先 运用差分GMM方法进行估计。作为一致估计, 差分GMM成立的前提是, 扰动项 $\left\{\varepsilon_{i t}\right\}$ 不存在自相 关。对此进行检验STATA Arellano-Bond 检验发现，一阶扰动项的差分p值为 0.0000 ，二阶扰 动项的差分 $\mathrm{p}$ 值为 0.1386 , 故扰动项的差分存在一阶自相关, 但不存在二阶自相关, 故接受原 假设 “扰动项 $\left\{\varepsilon_{i t}\right\}$ 无自相关”，可以使用差分GMM。作为比较, 本文运用系统GMM方法同样估 计以上模型。差分GMM和系统GMM的系数估计值与标准差比较如表 2 所示。

从表 2 可知, 差分GMM与系统GMM的系数估计值比较接近, 但后者的标准差更小, 因此本文 认为系统GMM估计更准确些。但系统GMM的有效性有待于检验。首先对系统GMM扰动项的自相关 性进行检验。STATA Arellano-Bond 检验结果表明, 一阶扰动项的差分p值为 0.0000 , 二阶扰 动项的差分 $\mathrm{p}$ 值为 $0.0944>0.05$, 故扰动项的差分存在一阶自相关, 但不存在二阶自相关, 故 $5 \%$ 显著性水平上接受 “扰动项差分的二阶自相关系数为 0 的假设” , 可以使用系统GMM。为了 进一步确认系统GMM估计的有效性, 我们运用过度识别检验, 结果p值 $=0.0830>0.05$, 接受 “所 有工具变量都有效” 的原假设。这表明系统GMM估计方法在此适用。由差分GMM估计与系统GMM 估计的比较可知, 本文选择更有效的系统GMM估计作为动态面板数据模型的估计方法。

2.4. 两种模型估计结果的比较

将动态面板数据与静态面板数据估计结果相比，得出以下结果：

(1) 由系统GMM估计结果可知，前一期人力资本质量积累水平对当期有显著的正效应， 前一期人力资本质量积累水平提高 1 个百分点, 则当期人力资本质量积累水平提高 0.7883 个百 
分点。（2）职工平均工资提高1\%带来的集群人力资本质量积累水平的提高幅度由原先的 $0.4478 \%$ 减弱为 $0.2927 \%$ 。（3）人均教育支出增加 $1 \%$, 则软件园人力资本质量积累水平提高 0.2398 个百分点, 稍高于固定效应估计结果。（4）产业集群规模扩大 $1 \%$, 软件园人力资本质 量积累水平下降幅度从原先的 $0.6761 \%$ 减弱为 $0.6319 \%$ 。

表2 产业集群人力资本质量积累水平决定因素估计（动态面板数据模型）

\begin{tabular}{c|c|c}
\hline \multirow{2}{*}{ 产业集群外部环境因素 } & 差分GMM & 系统GMM \\
\hline \multirow{2}{*}{$L 1 . \ln y_{\mathrm{it}}$ （滞后一期） } & $0.7883376 *$ & $0.3190508 *$ \\
\cline { 2 - 3 } & 0.5447880 & 0.2307388 \\
\hline \multirow{2}{*}{$\ln s a l$} & $0.1666261 * * *$ & $0.2927278 * * *$ \\
\cline { 2 - 3 } & 0.4051746 & 0.2646846 \\
\hline \multirow{2}{*}{ doct } & 0.0016866 & 0.0006194 \\
\cline { 2 - 3 } & 0.0019903 & 0.0023344 \\
\hline \multirow{2}{*}{$\ln e d u$} & $0.3607825 *$ & $0.2397902 * *$ \\
\cline { 2 - 3 } & 0.1881168 & 0.1122787 \\
\hline \multirow{2}{*}{ grn } & -0.0021046 & 0.0012517 \\
\cline { 2 - 3 } & 0.0018098 & 0.0022598 \\
\hline \multirow{2}{*}{} & -0.0181393 & 0.0020775 \\
\cline { 2 - 3 } & 0.0165503 & 0.0091303 \\
\hline
\end{tabular}

产业集群自身因素

\begin{tabular}{c|c|c}
\hline \multirow{2}{*}{ gov } & 0.1027121 & -0.028846 \\
\cline { 2 - 3 } & 0.2476256 & 0.1392134 \\
\hline \multirow{2}{*}{$\ln$ clus } & 0.0765254 & 0.1633435 \\
\cline { 2 - 3 } & 0.1413833 & 0.1002356 \\
\hline \multirow{2}{*}{$\ln$ scal } & $-1.057270 * * *$ & $-0.6318544 * * *$ \\
\cline { 2 - 3 } & 0.2439414 & 0.1603267 \\
\hline 常数项 & $18.360684 * *$ & $12.358972 * * *$ \\
\hline & 8.1699954 & 3.465099 \\
\hline
\end{tabular}

注：系数下方的值为标准差， $* * *$ 表示在 $1 \%$ 水平上显著，**表示在 $5 \%$ 水平上显著，*表示在 $10 \%$ 水平上显著。

\section{3. 结论与建议}

根据以上模型分析结果, 给出如下的结论与建议:

(1) 全国各软件园来说，应注重初始人力资本质量积累，后续的积累则会在初始惯性推 动下不断提高。（2）软件园所在城市政府相关部门应切实提高职工工资水平，以吸引外围人 力资本流入，从而提高软件园人力资本质量积累水平。

\section{References}

[1] Huang Chongli, Peng Zhenglong. Study of human capital multiplication based on system dynamics.Beijing: Science Press, 2012.

[2] Ministry of science and Technology Torch High Technology Industry Development Center. CHINA TORCH STATISTICAL YEARBOOK [M].Beijing:China Statistical Press, 2009-2012.

[3] Bureau of urban social and economic research, National Bureau of Statistics. CHINA CITY STATISTICAL YEARBOOK [M]. Beijing:China Statistical Press,2009-2012. 